\title{
Incidence and Risk Factors of Surgical Site Infections in Urological Surgery at Brazzaville Teaching Hospital
}

\author{
A. M. Ondongo Atipo, S. A. Ondziel, A. W. S. Odzébé*, M. R. Banga, Y. Lere, Y. Dimi, P. A. Bouya \\ Urology-Andrology Department, University Hospital of Brazzaville, Brazzaville, Republic of the Congo \\ Email: *odzebe_s@yahoo.fr
}

How to cite this paper: Atipo, A.M.O., Ondziel, S.A., Odzébé, A.W.S., Banga, M.R., Lere, Y., Dimi, Y. and Bouya, P.A. (2019) Incidence and Risk Factors of Surgical Site Infections in Urological Surgery at Brazzaville Teaching Hospital. Open Journal of Urology, 9, 42-50.

https://doi.org/10.4236/oju.2019.92005

Received: November 13, 2018

Accepted: February 24, 2019

Published: February 27, 2019

Copyright $\odot 2019$ by author(s) and Scientific Research Publishing Inc. This work is licensed under the Creative Commons Attribution International License (CC BY 4.0).

http://creativecommons.org/licenses/by/4.0/

cc) (i) Open Access

\begin{abstract}
Aim: To evaluate the incidence of surgical site infection in the Urology Department of Brazzaville teaching hospital. Methods: This was a prospective study performed in the Urology Department and operating room of the Brazzaville teaching hospital during six months from February $2^{\text {nd }}$ to July $2^{\text {nd }}$ 2017. The study concerned all patients who had undergone surgery, selected during the operating program and those who had been in emergency and then hospitalized in the urology department. These patients were followed for one month after the intervention date. Results: 209 patients were operated on of whom 48 had surgical site infection, a cumulative incidence of $22.96 \%$. The average age of infected patients was 58.3 years \pm 17.73 ds (extremes from 13 to 85 years). $43.75 \%$ infected patients had co-morbidity factors. The SSI rate was $70 \%$ in patients with positive urine culture. Urinary catheters were found in $33.33 \%$ of patients. The average length of preoperative hospital stay was two days. The rate of SSI in patients classified Asa I was $10.41 \%$, Asa II $37.5 \%$ and Asa III $52.09 \%$. The SSI rate was respectively $5.21 \%, 56.76 \%$ and $38.03 \%$. The infected patients operated first in the operative program accounted for $10.42 \%$. The group of patients who underwent prostatic surgery accounted for $42.58 \%$ of patients with an SSI rate of $47.91 \%$. The practice of aseptic measures by staff was found in $70 \%$ of cases. The infection rate in patients with drain was $73.23 \%$. The infection rate in patients with catheters was $54.26 \%$. The SSI was superficial in $66.67 \%$ of cases, deep in $25 \%$ of cases and organ in $8.33 \%$ of cases. Escherichia coli was the most frequently isolated germ 50\%. Conclusion: Surgical site infection (ISO) is a common feature in our practice. The advanced age of patients and comorbidity factors are associated with a high risk of occurrence of SSI. These infections were not inevitable, their incidence can be greatly reduced by specific preventive measures.
\end{abstract}




\section{Keywords}

Surgical Site Infection, Urology, Brazzaville Teaching Hospital

\section{Introduction}

Formerly known as wound infections, post-operative infections are defined as an infection occurring on the operative wound within 30 days after the procedure, or in the year in case of placement of a prosthesis or an implant [1].

Often easy to diagnose, they constitute not only the major cause of postoperative morbidity and mortality for the patient, but also an economic burden for the hospital.

Surgical site infections (SSI) account for $11 \%$ of all nosocomial infections (C. Clin Paris Nord). They constitute the second cause of nosocomial infections after urinary tract infection [2]. They affect $3 \%$ to $7 \%$ of the operated, their median time of onset is ten days after surgery. Americans estimate their prevalence between $4.12 \%$ and $16.5 \%$ [3]. Morbidity, mortality, disability, length of hospital stay and increased cost of hospitalization make this complication a public health problem around the world.

Urologic surgery occupies an important place in the surgical activity in general thanks to the progress and the development of new technologies. It uses probes and drains, which are important risk factors in the occurrence of surgical site infections.

In developed countries, national data are available to promote the way for the establishment of epidemiological surveillance and for taking of preventive measures. However, very few developing countries have statistical data on surgical site infections. In Congo, as in most sub-Saharan countries, very few studies on urology site infections have been performed to update this issue. Thus, we undertook to carry out this initial study which the main purpose was to evaluate the incidence of this infection in the urology department of Brazzaville teaching hospital.

\section{Methods}

This was a prospective study, carried out in the urology-andrology department and in the operating theater of the of Brazzaville teaching hospital during the six-month period from 2 February to 2 July 2017.

The work included all patients who had undergone surgery, who had been selected during the operating program, and those who had undergone emergency surgery and had been hospitalized in the urology department. These patients were followed for one month after the intervention date. All no operate patients were not included in patients without surgery were excluded from this study.

An infection of the operating site was defined by that occurring within 30 days (if no prosthesis in place) or in the year (if prosthesis in place) following the in- 
tervention. The diagnostic criteria are those validated by CDC D'ATLANTA USA [4] 1 taken up by CLIN in France:

- Infection of the superficial part of the incision, it affects the skin or mucous membranes, subcutaneous tissues or tissue located above the fascia of lining,

- Infection of the deep part of the incision, concerns deep soft tissues (fascia, muscles),

- Infection of the organ or space concerned by the operative site, it affects the organ or the space of the operating site (any anatomical part, other incision, opened or manipulated during the intervention).

The collection of data was made from a survey sheet, based on the patient's medical record and the results of the daily examination both pre- and postoperatively.

The variables studied were epidemiology (age, occupation, marital status) preoperative situation (preoperative shower taking by the patient, shaving of the patient, preoperative uroculture, urinary catheter port, ASA type, preoperative hospital stay duration). Operative situation (disinfection of the operating room, patient's row in the operative program, number of the nursing staff in the block, wearing of cap and bib, practice of rules of asepsis by the staff, type of anesthesia), postoperative situation (drain type, probe port, date of onset of fever, type of suppuration, date of onset of infection, pus sampling results, treatment, duration of son, postoperative duration).

The results were presented as mean and standard deviation for the quantitative variables and as a percentage for the qualitative variables. All information was entered and recorded on a pre-established Excel computer form, and all analyzes were performed using the Epi info 7 software. The test significance threshold was chosen at 0.05 .

\section{Results}

\subsection{Epidemiology}

During the study period, 209 patients were operated on, 7 of whom were emergency patients. 48 had surgical site infection, a cumulative incidence of $22.96 \%$.

The average age of the entire population was $48.13 \pm 20.55 \mathrm{SD}$, with extremes ranging from 2 to 85 years. The average age of infected patients was significantly higher at 58.3 years $\pm 17.73 \mathrm{ds}(\mathrm{p}>0.001)$ with extremes ranging from 13 to 85 years.

There were 38 men (79.17\%) and 10 women (20.83\%), a sex ratio of 3.8 for infected patients and 6.66 for uninfected patients. We found $31.25 \%$ of civil servants, 14.60 of shopkeepers, $16.66 \%$ of workers, $10.40 \%$ of students and $27 \%$ of unemployed patients. Married patients were found in $56.25 \%$ of cases, widowers in $12.5 \%$ of cases and singles in $31.25 \%$ of cases.

Co-morbidity factors were found in 33 patients $(15.78 \%)$ in the general population. Among the infected patients 21 had a co-morbidity factor of $43.75 \%$. There were 5 diabetic patients (23.80\%), 8 hypertensive patients (38.09\%) and 11 
patients with renal insufficiency (52.38\%).

\subsection{Preoperative Situation}

The preoperative shower taken by the patient the day before surgery was found in $66.6 \%$ of cases and the day of the intervention in $33.33 \%$ of cases. The shaving of the patient with the razor blade was done in $73 \%$ of the cases the day before and the morning of the day of the intervention in $27 \%$. Preoperative uroculture was performed in $80 \%$ of patients before the procedure. It was positive in $30 \%$ of infected patients. The ISO rate was $70 \%$ in patients with positive urine culture.

Urinary catheters were found in $33.33 \%$ of patients. The average duration of pre-operative hospitalization was two days, with extremes ranging from 1 to 7 days.

The evaluation of the ASA Score in the general population gave the following distribution, Asa I 52 patients (24.88\%), Asa II 65 patients $(31,10 \%)$ and Asa III 92 patients (44.01\%). The rate of SSI in patients classified Asa I was 10.41\%, Asa II $37.5 \%$ and Asa III 52.09\%.

Surgery contamination class (Altmeier classification) was clean in $22.91 \%$ of cases, clean contaminated in $70.84 \%$ and Dirty in $6.25 \%$. The SSI rate was respectively 5.21\%, 56.76\% and 38.03\%. Patients with index 2 and 3 had an incidence of $41.9 \%$ (Table 1 ).

\subsection{Intraoperative Situation}

The disinfection of the operating room was done after each intervention by a wet wash with bleach. Among infected patients, those operated first in the operating program accounted for $10.42 \%$, those in the second row for $33.33 \%$ and those in the third row for $56.25 \%$. The number of nursing staff in the block was on average 6 with extremes of 4 and 9. The wearing of the cap and bib was systematic in all cases. The practice of aseptic measures by staff was found in $80 \%$ of cases.

Anesthesia was general in $37.50 \%$ of cases and regional by spinal anesthesia in $62.5 \%$ of cases. Disinfection of the operative field was done with polyvidone iodine in all patients. The group of patients who underwent prostatic surgery accounted for $42.58 \%$ of patients with an SSI rate of $47.91 \%$ followed by those for renal disease $14.35 \%$ with an SSI of $18.75 \%$ (Table 2). The average duration of intervention was 58.57 minutes with extremes ranging from 30 to $190 \mathrm{mi}$ nutes.

Table 1. SSI incidence rate according to the NNIS score.

\begin{tabular}{cccc}
\hline Index NNIS & Number of Interventions & Number of SSI & Rate per 100 Operated (\%) \\
\hline 0 & 73 & 8 & 10.9 \\
1 & 105 & 27 & 25.7 \\
2 et 3 & 31 & 13 & 41.9 \\
Total & 209 & 48 & 22.9 \\
\hline
\end{tabular}


Table 2. Distribution of the general population and patients who presented the infection of the operative site according to the organ operated.

\begin{tabular}{ccccc}
\hline \multirow{2}{*}{ Operated organ } & \multicolumn{2}{c}{ General population } & \multicolumn{2}{c}{ Patient with SSI } \\
\cline { 2 - 5 } & Effectif & Percentage & Effectif & Percentage \\
\hline Spermatic cord & 28 & 13.39 & 1 & 2.08 \\
Penis & 1 & 0.47 & - & - \\
Testis & 14 & 6.69 & 1 & 2.08 \\
Prostate & 89 & 42.58 & 23 & 47.91 \\
Kidney & 30 & 14.35 & 9 & 18.75 \\
Urethra & 17 & 8.13 & 7 & 14.58 \\
Bladder & 18 & 8.60 & 4 & 8.33 \\
Bladder + vagina & 11 & 5.30 & 3 & 6.25 \\
Vulva & 1 & 0.47 & - & - \\
Total & 209 & 100 & 48 & 100 \\
\hline
\end{tabular}

\subsection{Postoperative Situation}

Patients carrying the drain accounted for $81.25 \%$ of cases. The rate of infection in patients with drain was $73.23 \%$ and $16.12 \%$ in non-carriers. The average drain wearing time was 3.55 days $\pm 1.04 \mathrm{~d}$ with extremes of 2 and 7 days. Urinary catheters were found in $64.58 \%$ of patients. The infection rate in patients with catheters was $54.26 \%$ and $13.40 \%$ in non-carriers.

The average time to onset of fever was 55.26 hours \pm 26 ds with extremes of 3 hours and 96 hours ( 4 days). The SSI was superficial in $66.67 \%$ of cases, deep in $25 \%$ of cases and organ in $8.33 \%$ of cases. The average time to onset of infection was $3 \pm 0.75$ days with extremes of 3 and 7 days.

The cultivation of pus was carried out in all cases. Escherichia coli was the most commonly isolated organism 50\%, followed by Staphyloccus aureus $16.67 \%$ (Table 3).

The susceptibility of germs to antibiotics during pus cultivation was as follows:

- Escherichia coli more susceptible to ceftriaxone, ciprofloxacin and less susceptible to norfloxacin and cefotaxime.

- Staphylococcus aureus and pseudomonas sensitive to clavulanic acid + amoxicillin.

- Klebsiella pneumonia, susceptible to chloramphenicol, colimycin, Amikacin, cotrimoxazole.

- Streptococci, sensitive to ceftriaxone and ciprofloxacin.

The mean duration of cutaneous sutures before removal was 10,016 $\pm 21,627$ ds with extremes ranging from 10 to 18 days. The duration of the postoperative hospital stay was less than 15 days in $25 \%$ of cases, between 15 and 20 days in $66.67 \%$ of cases and between 21 and 24 days in $8.33 \%$ of cases. Mean postoperative hospital stay was 12 days with extremes of 10 to 24 in infected patients. 
Table 3. Distribution of patients according to the type of germs.

\begin{tabular}{ccc}
\hline Type of germs found in the culture of the pus & Effective & Percentage \\
\hline Escherichia coli & 24 & 50 \\
Staphylocoque aureus & 8 & 16.67 \\
Klebsiella pneumonia & 5 & 10.42 \\
Entérobactérie & 6 & 12.5 \\
Pseudomonas aeroginosa & 3 & 6.25 \\
Streptococcus & 2 & 4.16 \\
Total & 48 & 100 \\
\hline
\end{tabular}

Antibiotic prophylaxis was performed in $89.58 \%$ of all operated patients. The rate of SSI was $57 \%$ in patients who received antibiotic prophylaxis.

Of the 209 patients who underwent surgery, all patients were seen at the fifteenth and thirtieth day after hospitalization.

\section{Discussion}

The impact of SSI is variously reported in the literature for developed countries, where nosocomial infection control mechanisms [5] are established, and non-developed countries where they are almost non-existent. Thus, our results are similar to those of DEMBELE, which found a $23.63 \%$ incidence in the urology department of the teaching hospital of the G [6] in MALI, Togo et al. reported a higher frequency of $65.60 \%$ [7]. The incidence of SSI is much lower in the United States and Europe, ranging from $1.77 \%$ to $7 \%$ [3] [8] [9]. The high incidence of SSI in our study can be explained not only by the absence of a national health policy for the control of nosocomial infections, but also by the presence of risk factors for the occurrence of surgical site infections in our sample.

The high age increases the risk of SSI due to the weakening of the immune state and the loss of the skin barrier. Patients with a high elderly were the most numerous in our study, which is certainly related to a large number of patients operated for benign prostatic hypertrophy. This result is close to that of Fourcade [10] in France and KAMBO in Burkina Faso [11].

Diabetes, arterial hypertension and renal failure were co-morbidity factors associated with a high risk of site infection [12] [13]. Many other risk factors are found in the literature such as: obesity, intraoperative hyperglycemia, smoking, malnutrition, anemia, inadequate practice of care.

More than half of the patients concerned were ASA II and ASA III patients. According to the literature, the level of SSI increased according to the pre-anesthetic score (ASA) and the Altemeier contamination class [8].

Preoperative duration of stay is an important preoperative risk factor because of the change in microbial flora of the cutaneous and digestive tract by the third day [8]. She was important in our series with an extreme of seven days. It is 
ideally accepted that patients should be interned the day before or the morning of surgery [14].

Antibiotic prophylaxis is a very effective means of fighting post-operative infections. $89.58 \%$ of our patients had received antibiotic prophylaxis. This practice alone has not reduced the incidence rate of SSI. That's why it must be combined with preventive hygiene measures for high efficiency. Antibiotic prophylaxis is not devoid of consequences such as increasing resistance and changing the bacterial flora of patients. Strict rules must govern its use, such as: respect for indications and the choice of molecules. This prophylaxis must begin before surgery and cover the entire duration of the procedure until closure, without ever exceeding 24 hours.

Almost all of our interventions involved the prostate, kidney and bladder. The essential procedures on these organs require the use of probes and drain, which are foreign bodies constituting a risk factor in the occurrence of SSI. Abreu D. et al. [15], in a series of 70 patients undergoing prostatic adenomectomy, surgical site infections were noted in $18 \%$ of cases. Surgery on the urinary tract, especially when urine was infected, was a risk factor for surgical site infections.

The duration of the intervention has been identified as a risk factor in several studies [16]. This risk is significant in interventions that last more than two hours. [14] More than half of the interventions in this study lasted less than one hour.

It should be noted that the onset of fever on the first and second day of the procedure does not always indicate suppuration of the operative site, it may be related to inflammation, resorption of red blood cells, dehydration or other associated pathologies.

In our study $66.67 \%(\mathrm{n}=32)$ of patients had superficial suppuration. FARTHOUAT [17] in Dakar reported 1 case out of 10 patients, a percentage of $10 \%$. The high rate of superficial suppuration found in our study can be explained by the fact that the majority of our patients were operated for prostatic pathologies requiring the establishment of an indwelling catheter, which constitutes a foreign body favoring the colonization of germs therefore infection suppuration type of the operative wound. The majority of patients had suppurated between the third $39.60 \%(n=19)$ and the fourth $27.08 \%(n=13)$ day. These results are not consistent with those of DEMBELE who reported $61.01 \%$ between the seventh and tenth day [6].

\section{Conclusion}

Surgical site infection (SSI) is a common feature in our practice. The advanced age of patients and comorbidity factors (diabetes, hypertension, renal failure) are associated with a high risk of occurrence of SSI. The delay of occurrence of the SSI in our series is short and can be explained by the non-respect of the rules of perioperative asepsis, the inexperience of the personnel in the operating room, the lack of self management sterilization procedures, the lack of cytobacteriolog- 
ic examination process of the urine 48 hours before the intervention and the lack of antibiotic prophylaxis within the required time. These infections were not inevitable, their incidence can be greatly reduced by specific preventive measures. This prevention is based on the implementation of a national policy for the control of nosocomial infections and scrupulous observation of hospital hygiene measures.

\section{Approved}

This study has been approved by the Ethics Committee.

\section{Funding}

We did not receive any funding.

\section{Conflicts of Interest}

We declare that we have no conflicts of interest with this article.

\section{References}

[1] Horan, T.C., Gagnes, R.P., Martone, W.J., Jarvis, W.R. and Emori, T.G. (1992) CDC Definitions of Nosocomial Surgical Wound Infections. American Journal of Infection Control, 20, 271-274. https://doi.org/10.1016/S0196-6553(05)80201-9

[2] Perniceni, T. and Vons, C. (2002) Interests of a Program to Monitor the Incidence of Surgical Site Infections in Digestive Surgery. Annales de Chirurgie, 127, 786-790. https://doi.org/10.1016/S0003-3944(02)00918-5

[3] De Lissovoy, G., Fraeman, K., Hutchins, V., Murphy, D., Song, D. and Vaughn, B.B. (2009) Surgical Site Infection: Incidence and Impact Utilization and Treatment Costs. American Journal of Infection Control, 37, 387-397. https://doi.org/10.1016/j.ajic.2008.12.010

[4] CDC (2011) Surgical Site Infection (SSI) Event.

[5] National Agency for Accreditation and Evaluation in Health (2003) Nosocomial Infections: How to Interpret Rates? The Example of Surgical Site Infections. Report, $118 \mathrm{p}$.

[6] Dembelé, A., et al. (2006) Surgical Wound Infections in the Urology Department of the HNP Teaching Hospital (Bamako). Médecine d Afrique Noire, 53, 402-405.

[7] Togo, A., Coulibaly, Y., Dembélé, B.T., Togo, B., Keita, M., Kanté, L., et al. (2011) Risk Factors for Surgical Site Infection in Children at the Teaching Hospital Gabriel Touré Bamako. Journal of Hospital Infection, 79, 371-372. https://doi.org/10.1016/j.jhin.2011.08.007

[8] Surveillance of Surgical Site Infections in France in 1999 and 2000 Results Report. 2003.

[9] Astagneau, P., Daniel, F., Olivier, M., et al. (2010) Surveillance of Surgical Site Infection in France: Experience of a Network during the Last Decade. Symposium HAS-BMJ-19 Avril, Nice.

[10] Fourcade, R.O., et al. (1997) Prostate. Ed John Libbey Euro, Paris, 162 p.

[11] Kambou, T., Zango, B., et al. () Surgical Treatment of Benign Prostatic Hypertrophy at the Teaching Hospital SANOU de BOBO Dioulasso. Médecine d Afrique Noir, 
29, 606-612.

[12] Korol, E., Johnston, K., Waser, N., Sifakis, F., Jafri, H.S., Lo, M., et al. (2013) A Systematic Review of Risk Factors Associated with Surgical Site Infections among Surgical Patients. PLoS ONE, 8, e83743. https://doi.org/10.1371/journal.pone.0083743

[13] Ngaroua, Ngah, J.E., Bénet, T. and Djibrilla, Y. (2016) Incidence of Surgical Site Infections in Sub-Saharan Africa: Systematic Review and Meta-Analysis. Pan African Medical Journal, 24, 171. https://doi.org/10.11604/pamj.2016.24.171.9754

[14] Francioli, P., Nahimana, I., Lausanne and Bâle, W.A. (1996) Surgical Site Infections: Review. $S N, 3,1$.

[15] Abreu, D., Campos, E., Seija, V., Arroyo, C., Suarez, R., Rotemberg, P., et al. (2014) Surgical Site Infection in Surgery for Berign Prostatic Hyperplasia: Comparaison of Two Skin Antiseptics and Risk Factors. Surgical Infection, 15, 763-767.

[16] Desjeux, G., Pascal, B., Faucompret, S., Brissiaud, J.C., Perrier, C., Thierry, H., et al. (2000) Incidence of Surgical Site Infections in a Visceral Surgery Department. Medecine et Armee, 28, 25-29.

[17] Farthouat, P.H., et al. (2009) Surgical Site Infections in Visceral Surgery. Médecine d Afrique Noire, 3, 143-147. 